\title{
Heavy-ion transfer reactions at large internuclear distances using the PRISMA magnetic spectrometer
}

\author{
D. Montanari ${ }^{1, a}$, L. Corradi ${ }^{2}$, S. Szilner ${ }^{3}$, G. Pollarolo ${ }^{4}$, E. Fioretto ${ }^{2}$, A.M. Stefanini ${ }^{2}$, E. Farnea ${ }^{1,5}$, C. Michelagnoli ${ }^{1,5}$, \\ G. Montagnoli ${ }^{1,5}$, F. Scarlassara ${ }^{1,5}$, C.A. Ur ${ }^{1,5}$, S. Courtin ${ }^{6}$, A. Goasduff ${ }^{6}$, F. Haas ${ }^{6}$, T. Mijatović ${ }^{3}$, and N. Soić ${ }^{3}$ \\ ${ }^{1}$ Istituto Nazionale di Fisica Nucleare, Sezione di Padova, I-35131 Padova, Italy \\ ${ }^{2}$ Istituto Nazionale di Fisica Nucleare, Laboratori Nazionali di Legnaro, I-35020 Legnaro, Italy \\ ${ }^{3}$ Ruđer Bošković Institute, HR-10 002 Zagreb, Croatia \\ ${ }^{4}$ Dipartimento di Fisica Teorica, Università degli Studi di Torino, and Istituto Nazionale di Fisica Nucleare, I-10125 Torino, Italy \\ ${ }^{5}$ Dipartimento di Fisica e Astronomia, Università degli Studi di Padova, I-35131 Padova, Italy \\ ${ }^{6}$ Institut Pluridisciplinaire Hubert Curien, CNRS-IN2P3, Université de Strasbourg, F-67037 Strasbourg, France
}

\begin{abstract}
We measured excitation functions for the main transfer channels in the ${ }^{116} \mathrm{Sn}+{ }^{60} \mathrm{Ni}$ reaction at different bombarding energies from above to well below the Coulomb barrier. The experiment has been performed in inverse kinematics, detecting the lighter (target-like) ions with the magnetic spectrometer PRISMA at very forward angles. Good mass, nuclear charge and kinetic energy resolutions have been achieved. The comparison between the data and microscopic calculations for the present case and for the previously measured ${ }^{96} \mathrm{Zr}+{ }^{40} \mathrm{Ca}$ system, namely superfluid and near closed shells nuclei, should significantly improve our understanding of nucleon-nucleon correlation properties in multinucleon transfer processes.
\end{abstract}

\section{Introduction}

Transfer reactions at energies close to the Coulomb barrier always played an important role for nuclear structure and reaction dynamic studies. In heavy ion transfer reactions many channels become available at the same time thus giving the possibility to study the role played by single and pair transfer modes [1]. In literature most of the studies with heavy ions involve measurements of inclusive cross-sections at and above the Coulomb barrier, where the theoretical interpretation is particularly difficult due to the presence of many competing processes. On the contrary, at bombarding energies below the Coulomb barrier and at large internuclear distance, nuclei interact through the tail of their density distribution and are only slightly influenced by the nuclear potential. Under these conditions, reaction products are excited in a limited energy region (few $\mathrm{MeV}$ ), as shown in [2] where at low bombarding energies the TKEL spectra have a width of $\approx 3 \mathrm{MeV}$, therefore the complexity of coupled channel calculations diminishes, since few populated excited states have to be taken into account, and more quantitative information may be extracted on particle correlations [3]. Traditionally, transfer probabilities $[4,5], P_{t r}$ 's (ratio of the transfer over the elastic cross sections), have been extracted measuring excitation functions at fixed angles or angular distributions at fixed bombarding energies. The $P_{t r}$ obtained with the two methods should coincide if ions interact at very large distances, where deep inelastic components drop off. In most

\footnotetext{
a e-mail: daniele.montanari@pd.infn.it
}

of the previous works $P_{t r}$ 's have been derived from angular distributions at energies above the Coulomb barrier, where either diffractive effects [6] or the presence of reaction mechanisms other than the direct one, i.e. two-step process, [7] may contribute to cause slope anomalies . Excitation functions at energies below the barrier have been performed in only a few cases [8-11], due to experimental difficulties and the limitation in detection efficiency. Since a few years, using large acceptance magnetic spectrometers, it is possible to study multinucleon transfer reactions at energies far below the Coulomb barrier with good mass and atomic number resolution, especially in inverse kinematics conditions where the lighter target-like recoils have high kinetic energy. The efficient use of the large solid angle spectrometer PRISMA to perform such studies has already been demonstrated in the study of the ${ }^{96} \mathrm{Zr}+{ }^{40} \mathrm{Ca}$ system [2]. Making use of inverse kinematics, excitation functions for the most intense transfer channels have been obtained by detecting target recoils and varying the bombarding energies in several steps from above to well below the barrier, $0.75 \leq \mathrm{E} / \mathrm{V}_{B} \leq 1.05$, being $\mathrm{V}_{B}=475 \mathrm{MeV}$ in the lab system, corresponding to a broad range of distances of closest approach. The data have been compared with microscopic calculations performed within a semi-classical theory $[12,13]$, where the two particle transfer included the $0^{+}$states of both projectile and target. From the comparison, the role played by the $\mathrm{f}_{7 / 2}$ and $\mathrm{p}_{3 / 2}$ single particle states has been identified. While the first dominates the ground state wave function of ${ }^{42} \mathrm{Ca}$ the second dominates the wave function of the $0^{+}$state at $5.76 \mathrm{MeV}$. Such a state 
for the $+2 n$ channel has a good match with the optimum Q-value, being $Q_{g s}=+5.6 \mathrm{MeV}$ and $Q_{o p t} \approx 0 \mathrm{MeV}$. The importance of states with different spin and parity has been also evidenced. As a further step we found it interesting to investigate another system whose ground to ground state $Q$-value is close to zero for neutron transfers, matching their optimum Q-value. The ${ }^{60} \mathrm{Ni}+{ }^{116} \mathrm{Sn}$ system is very suitable in this sense, since it has $Q_{g s}^{+1 n}=-1.7 \mathrm{MeV}$ and $Q_{g s}^{+2 n}=+1.3 \mathrm{MeV}$. One then expects to have a main population close to the ground to ground state transitions and, in particular for the $+2 \mathrm{n}$ channel, it is interesting to see how calculations including only transfer to the $0_{g s}^{+}$states compare with the experimental data. We present below some preliminary results of a reaction involving the ${ }^{60} \mathrm{Ni}+{ }^{116} \mathrm{Sn}$ system in inverse kinematics performed using the magnetic spectrometer PRISMA [14-16].

\section{The experiment and experimental results}

Similarly to the ${ }^{96} \mathrm{Zr}+{ }^{40} \mathrm{Ca}$ case $[2,17]$, we detected the light target-like ions by setting the magnetic spectrometer at a very forward angle. The detected reaction products had at the same time enough kinetic energy, resulting in a good mass resolution, and a forward focused angular distribution which allowed a high detection efficiency. The ${ }^{116} \mathrm{Sn}$ beam was delivered by the heavy ion PIAVE injector and the ALPI superconducting booster of the Laboratori Nazionali di Legnaro with an average current of $\sim 2$ pnA onto a $100 \mu \mathrm{g} / \mathrm{cm}^{2}{ }^{60} \mathrm{Ni}$ target with a Cbacking of $15 \mu \mathrm{g} / \mathrm{cm}^{2}$. Ni-like recoils have been detected by PRISMA at $\theta_{l a b}=20^{\circ}$, corresponding to $\theta_{\text {c.m. }}=140^{\circ}$. The beam energy was varied in steps of $\simeq 10 \mathrm{MeV}$ from 500 to $395 \mathrm{MeV}$. Some furhter points have been measured making use of an $85 \mu \mathrm{g} / \mathrm{cm}^{2}$ thick C-foil placed in front of the $\mathrm{Ni}$ targets. This was done to optimize the time loss due to the change of energy with ALPI and allowed to degrade the ${ }^{116} \mathrm{Sn}$ beam by about $7 \mathrm{MeV}$. Measurement at two energies, $\mathrm{E}_{l a b}=220$ and $280 \mathrm{MeV}$, have been performed with the Tandem only, to have a precise energy reference. The obtained excitation function, from above to $\simeq 25 \%$ below the Coulomb barrier, ranged from $\simeq 12$ to $\simeq 16$ fm of distance of closest approach.

Two monitor detectors have been placed close to the target at angular positions $\left(\theta_{l a b}=55^{\circ}\right.$ and $\left.60^{\circ}\right)$ which ensured the detection of pure Rutherford scattered ${ }^{60} \mathrm{Ni}$ and ${ }^{12} \mathrm{C}$ ions. The identification of the reaction products in PRISMA is based upon an event-by-event reconstruction of the trajectories of the ions $[15,16]$ inside the spectrometer. We use positional information provided by the start [18] and focal plane [19] detectors of PRISMA and the equation of motion of a charged particle in the quadrupole and dipole magnetic fields to reconstruct the trajectories of the ions inside PRISMA. Trajectories are considered to be planar and the effects of the fringing fields of the magnets are taken into account by assigning an effective length to the optical elements of the spectrometer. This is done in order to have a fast off-line algorithm to be used for data analysis. Under these assumptions the trajectories are uniquely

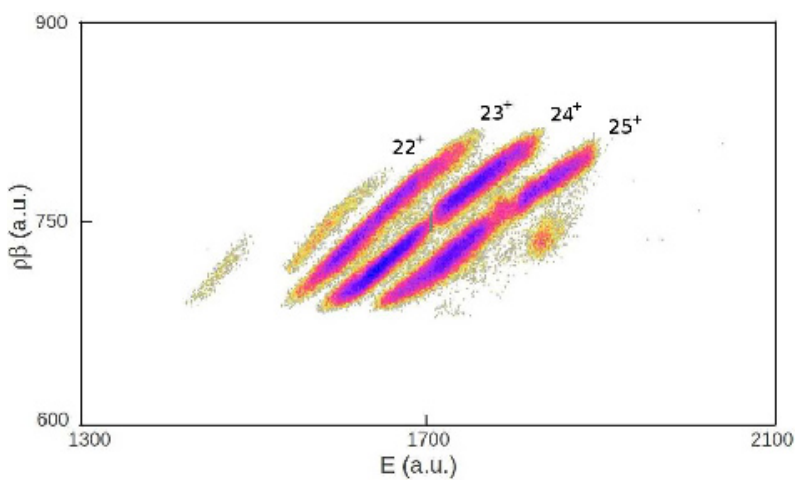

Figure 1. $\rho \beta$ versus $E$ matrix, constructed to select the different atomic charge states of Ni-like ions. The central structure in the matrix corresponds to the most probable charge state $q=24^{+}$. The assignment of the other $q$ values is given correspondingly.

defined by only one parameter, the bending radius $\rho$ in the dipole.

The assignment of the atomic number $\mathrm{Z}$ is obtained by using the energy loss $\Delta \mathrm{E}$ and the total kinetic energy $\mathrm{E}$ of the ions as provided by the ionization chamber. Due to the existence of several atomic charge states $q$, different $A / q$ values are obtained for each $Z$ and a selection of each single charge state is needed to construct the mass spectra. From the Lorentz equation it is possible to derive the proportionality $E \propto q \rho \beta$, with $\beta=v / c$, thus from a two-dimensional matrix $\rho \beta$ versus E one can select the atomic charge states, as shown in Fig. 1, where the separation among the different $q$ is clear. The reconstruction of the mass number $\mathrm{A}$, is based on the relation $A / q \propto B \rho / v$ which follows from the Lorentz equation, here $v$ is the velocity of the ion, which is given by $v=L / T O F$, being $L$ the length of the trajectory. All charge states are finally added together in order to obtain the mass spectra. Two mass spectra for Ni isotopes are displayed as examples in Fig. 2, showing the clear separation obtained for the different transfer channels. The two plots correspond to energies well above the Coulomb barrier $\left(\mathrm{E}_{l a b}=500 \mathrm{MeV}\right)$ and close to the barrier $\left(\mathrm{E}_{l a b}=468 \mathrm{MeV}\right)$. While in this last case only neutron pickup channels are present, as expected from simple optimum Q-value arguments, at the higher energy one sees the onset of neutron stripping channels, which are derived for the presence of complex mechanisms involving large energy losses and consequent neutron evaporation from the primary fragments [1, 20-22].

Assuming a binary reaction and imposing the conservation of linear momentum, we could reconstruct the total kinetic energy loss (TKEL) for the different reaction channels. In Fig. 3 we display the TKEL spectra for the elastic, the one $(+1 n)$ and the two $(+2 n)$ neutron pick-up channels at the bombarding energy of $\mathrm{E}_{l a b}=475 \mathrm{MeV}$, close to the Coulomb barrier. The elastic(+inelastic) peak has a width of $\sim 2 \mathrm{MeV}$, close to the expected experimental energy resolution. The vertical dashed lines indicate the position of the Q-value for the elastic scattering, $Q_{e l}=0$ (channel 100 in the figure), which is taken as a reference for all the other isotopes. TKEL distributions for neutron transfers 


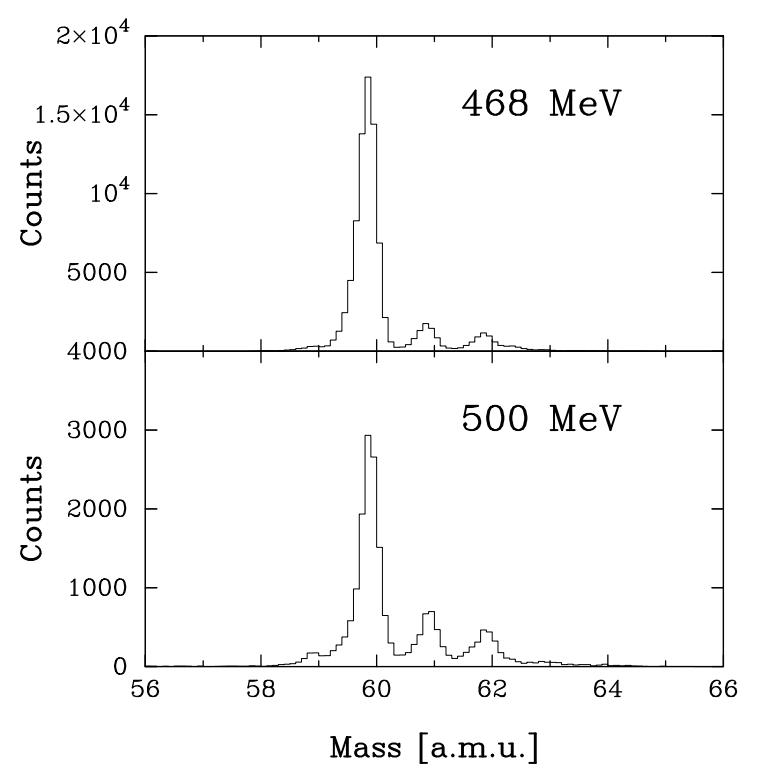

Figure 2. Mass spectra for two different bombarding energies for the ${ }^{116} \mathrm{Sn}+{ }^{60} \mathrm{Ni}$ reaction. We notice, for the highest beam energy, the onset of neutron stripping channels.

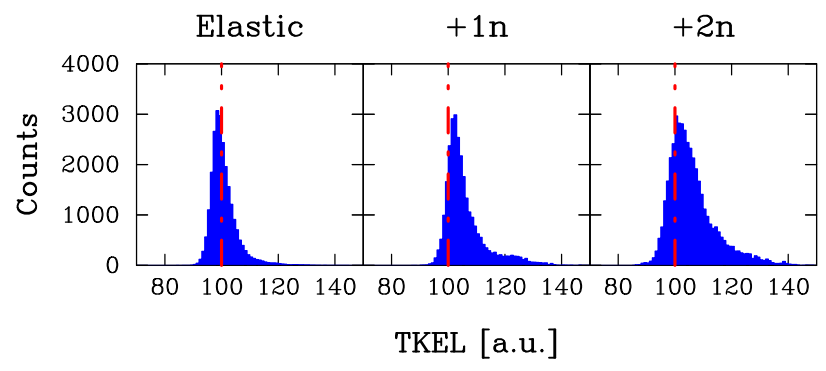

Figure 3. TKEL loss spectra for the elastic and $+1 \mathrm{n},+2 \mathrm{n}$ transfer channels at the bombarding energy $\mathrm{E}_{l a b}=475 \mathrm{MeV}$. See text for details.

are peaked around $Q=0$, as it is expected by considering that their ground to ground state Q-values are $Q_{g s}^{+1 n}=-1.7$ $\mathrm{MeV}$ and $Q_{g s}^{+2 n}=+1.3 \mathrm{MeV}$, respectively. At the same time one sees a tail toward larger TKEL, more marked for the $+2 n$ channel, typical of the energy regime close to the barrier. These energy loss components tend to disappear far below the barrier.

At present, data are being analyzed with the main aim to extract the transfer cross sections for the one and two neutron pick-up channels and for channels involving proton stripping. Relative normalization of the different runs has been obtained taking into account the geometry of monitors and of PRISMA. We assumed that for the different reaction channels the transport of the ions inside the spectrometer is constant. This is justified from the fact that Qvalue distributions for the different reaction channels are restricted to a small region well within the acceptance of the spectrometer. The absolute normalization is then obtained by assuming that well below the Coulomb barrier the elastic scattering corresponds to the pure Rutherford. The starting point in the analysis of the reaction mechanism is the study of the elastic scattering. We measured

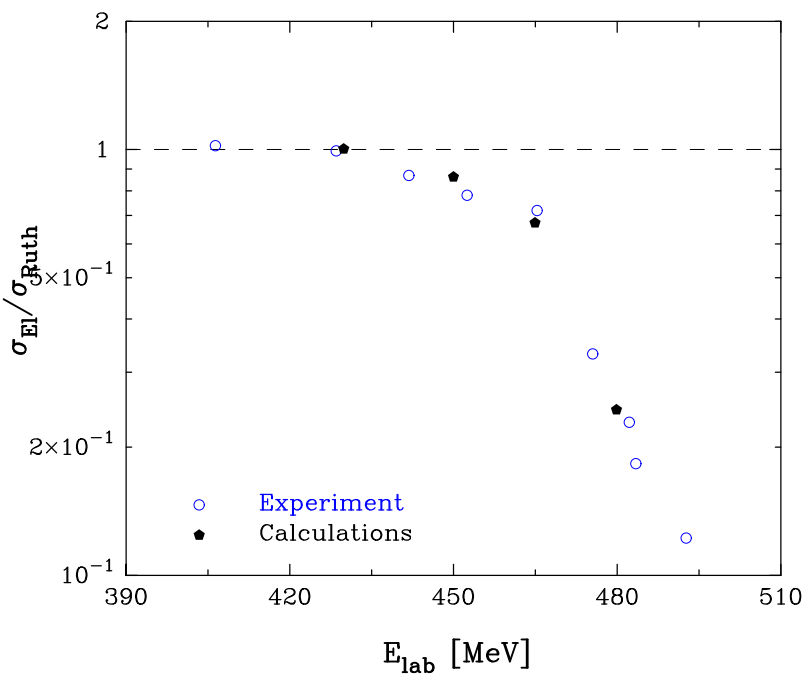

Figure 4. Ratio between the elastic cross section $\sigma_{e l}$ and the Rutherford cross section $\sigma_{\text {Ruth }}$. Full circles are the theoretical calculations, open circles represent the experimental values, with uncertainties smaller than the size of the symbols.

differential cross section for this channel and we plotted them in Fig. 4 as ratio over the pure Rutherford. As expected at very low energies the ratio is equal to one, while it starts decreasing when absorptive mechanisms take place. Data (open symbols) are compared with theoretical calculations (full symbols) by the semi-classical model GRAZING [23] showing a good agreement also in the higher energy region.

Transfer probabilities have been obtained as the ratio of differential cross sections of the transfer channels over the elastic. Angular distributions of the reaction products have almost the same shape for all the isotopes and are quite constant within the angular acceptance of the spectrometer. For this reason in order to minimize the statistical error we decided to integrate the full angular range and to assign the measured cross section to the central angle of PRISMA, which has also been used to calculate the distance of closest approach $D=\frac{Z_{1} Z_{2} e^{2}}{2 E_{c, m .}}\left(1+\frac{1}{\sin \left(\theta_{c, m} / 2\right)}\right)$.

Preliminary results for the transfer probabilities of one neutron, two neutron and one proton transfer channels are plotted as a function of D in Fig. 5 with open circles, full squares and triangles, respectively. Data are well described by an exponential function at big distances where the experimental elastic yield is almost completely due to pure Rutherford events. At higher energies (smaller distances) the slope of the experimental data deviates from the exponential trend due to the appearance of mechanisms other than the direct one. Proton stripping channels are in general more difficult to measure experimentally far below the barrier since they drop off more rapidly than neutron channels, therefore a more careful data analysis is mandatory. The $-1 p$ and the $+1 n$ channels are the basic building blocks for the understanding of the more complex multiple particle transfer. The comparison of their behaviour as a function of the bombarding energy will tell about the shape of the form factors $[2,24]$. 


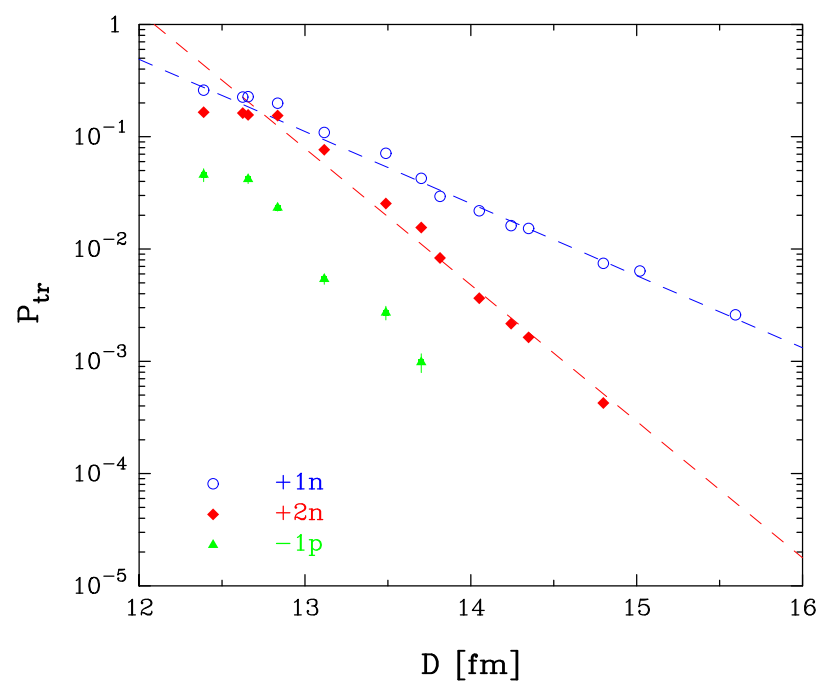

Figure 5. Transfer probabilities for $+1 \mathrm{n}$ (open circles), $+2 \mathrm{n}$ (full squares) and $-1 p$ (triangles) reaction channels plotted as a function of the distance of closest approach D. Neutron data extend down to $\simeq 15 \mathrm{fm}$, while for proton stripping further analysis is needed (see text for details). Errors are smaller than the size of the symbols for $+1 \mathrm{n}$ and $+2 \mathrm{n}$ channels.

\section{Conclusions}

We measured in inverse kinematics an excitation function for the ${ }^{116} \mathrm{Sn}+{ }^{60} \mathrm{Ni}$ system. Light target-like ions have been detected with the magnetic spectrometer PRISMA with a good mass and atomic number resolution in the whole energy range. Data analysis is in progress to deduce the cross sections and transfer probabilities for neutron pick-up channels as well as for channels involving proton stripping. The comparison between data and theory for the present case and for the previously measured ${ }^{96} \mathrm{Zr}+{ }^{40} \mathrm{Ca}$ system, namely superfluid and near closed shells nuclei, will significantly improve our understanding of nucleonnucleon correlations in the transfer process.

\section{Acknowledgements}

The authors wish to thank the LNL Tandem-ALPI stuff for providing us with the good quality beams and the target laboratory for the excellent target preparation. This work was partly supported by the European Community FP6 - Structuring the ERA - Integrated Infrastructure Initiative - contract EURONS No. RII3-CT-2004-506065. This work was, also, supported in part by the Croatian Ministry of Science, Education and Sports, Grant No. 0098-1191005-2890.

\section{References}

[1] L. Corradi, G. Pollarolo and S. Szilner, J. of Phys. G: Nucl. Part. Phys. 36, 113101 (2009).

[2] L. Corradi, S. Szilner, G. Pollarolo et al., Phys. Rev. C 84, 034603 (2011).

[3] B.F. Bayman and J.Chen, Phys. Rev. C26, 1509 (1982).

[4] R. Künkel, W. von Oertzen, H.G. Bohlen, B. Gebauer et al., Z. Phys. A 336, 71 (1990).

[5] W. von Oertzen, I. Peter, S. Thummerer, H. G. Bohlen, B. Gebauer et al., Eur. Phys. J. A 20, 153 (2004).

[6] A.H. Wuosmaa, K.E. Rehm, B.G. Glagola, Th. Happ, W. Kutschera, and F.L.H. Wolfs, Phys. Lett. B 255, 316 (1991).

[7] K.E.Rehm, B.G.Glagola, W.Kutschera, F.L.H.Wolfs, H.Wuosmaa, Phys. Rev. C 47 , 2731 (1993).

[8] R. R. Betts, P. M. Evans, C. N. Pass, N. Poffe et al., Phys. Rev. Lett. 59, 978 (1987).

[9] R. B. Roberts, S. B. Gazes, J. E. Mason, M. Satteson et al., Phys. Rev. C 47, R1831 (1993).

[10] C. L. Jiang, K. E. Rehm, Gehring J., Glagola B. et al, Phys. Lett.. B 337, 59 (1994).

[11] C. L. Jiang, K. E. Rehm, H. Esbensen et al., Phys. Rev. C 57, 2393 (1998).

[12] E.Maglione, G. Pollarolo, A. Vitturi, R. A. Broglia and A. Winther, Phys. Lett. B162, 59 (1985).

[13] J.H.Sorensen and A.Winther, Nucl. Phys. A 550, 306 (1992).

[14] A.M. Stefanini et al., Nucl. Phys. A701, 217c (2002).

[15] S. Szilner, C. Ur, L. Corradi, G. Pollarolo et al., Phys. Rev. C 76, 024604 (2007).

[16] D. Montanari, E. Farnea, S. Leoni, G. Pollarolo et al., Eur. Phys. J. A 47, 4 (2011).

[17] L. Corradi, S. Szilner, G. Pollarolo et al., Eur. Phys. J. Conf. Series 17, 08004 (2011).

[18] G. Montagnoli, A.M. Stefanini, M. Trotta et al, Nucl. Instrum. and Methods 547, 455 (2005).

[19] S.Beghini, L. Corradi, E.Fioretto et al, Nucl. Instrum. and Methods 551, 364 (2005).

[20] L. Corradi, A. M. Vinodkumar, A. M. Stefanini, E.Fioretto et al., Phys. Rev. C 66, 024606 (2002).

[21] S. Szilner, L. Corradi, G. Pollarolo, S. Beghini et al., Phys. Rev. C 71, 044610 (2005).

[22] S. Szilner, L. Corradi, F. Haas, D. Lebhertz et al., Phys. Rev. C 84, 014325 (2011).

[23] A. Winther, Nucl. Phys. A 594, 203 (1995).

[24] J.M. Quesada, G. Pollarolo, R. A. Broglia, A. Winther, Nucl. Phys. A 442, 381 (1985). 